\title{
Design of Intelligent Retrieval system for disordered surveying and Mapping Archives based on automatic Program
}

\author{
Yixin Chen ${ }^{1, a, ~}$, Ran $\operatorname{Yan}^{2, b}, \operatorname{Xinyv} \mathrm{Li}^{3, \mathrm{c}}$ \\ ${ }^{1}$ Xiamen University, School of Aerospace Engineering, Henan, Zhengzhou, 450000; \\ ${ }^{2}$ Zhengzhou University, School of Information Engineering, Henan, Zhengzhou, 450000; \\ ${ }^{3}$ Xiamen University, School of Aerospace Engineering, Shandong, Zibo, 255000; \\ ae-mail:chenyixin39@sina.com, be-mail: feiepuh@126.com, 'e-mail: 2639xs@sina.com
}

Keywords:automatic sequencing, unordered Mapping Archives, intelligent retrieval, network architecture, environment configuration, retrieval scripts

\begin{abstract}
The Tableau unordered surveying and mapping file retrieval system has some disadvantages, such as limited storage amount of resources, too long time of retrieval information export and so on. In order to solve the above problems, a new intelligent retrieval system of unordered surveying and mapping files based on automatic program is designed. The experimental results show that compared with the Tableau retrieval system, the upper limit of resource storage is increased by $2.5 \times 10^{11} \mathrm{~T}$ after the application of a new intelligent retrieval system of disordered surveying and mapping files based on automatic program. Retrieval information export time was shortened by 15 mins.
\end{abstract}

\section{Introduction}

The automatic program uses automatic means for program design and processing. At present, artificial intelligence logic (description logic) is mainly used for automatic program construction. Artificial intelligence logic is a common formal language tool. In the KL-ONE concept, the description logic is used to determine the logical attribute structure of the first-order predicate, and to generate a judgment subset with complete expression ability based on the actual judgment result. In order to quickly query electronic disordered mapping files, the traditional Tableau retrieval system, with the support of ALC basic theory, counts the total amount of electronic disordered mapping data to be encoded by expanding, adding time series operators and action operators. And the one-to-one correspondence between the mapping information in the database and the CTL-DDL automatic program are used, the intelligent retrieval processing of the disordered mapping file is completed, and the smooth operation of the system is realized ${ }^{[1-2]}$. However, as the system running time increases, the correspondence between the timing operator, the action operator and the CTLDDL automatic program begins to decline gradually. The results of simulation and comparison experiments show that the new intelligent retrieval system does have certain practical application value.

\section{Hardware design of intelligent retrieval system for disordered surveying and mapping files}

The hardware design process of the new intelligent retrieval system includes three basic links: automatic program dynamic network architecture design, intelligent retrieval module design, and disordered mapping storage module design. The specific construction methods are as follows.

\subsection{Automatic program dynamic network architecture design}

The automatic program dynamic network framework is the foundation for the new system hardware operating environment. According to the system's rules for the capture of unordered mapping data, the automatic program dynamic network framework has three main functions: retrieval condition determination, timing description logic specification, and action operator 
intelligent loop ${ }^{[3]}$. Therefore, the overall system hardware operating environment is still at rest ${ }^{[4-5]}$. The above operations are summarized to complete the dynamic network framework construction of the system automatic program. The specific framework structure is shown in Figure 1.

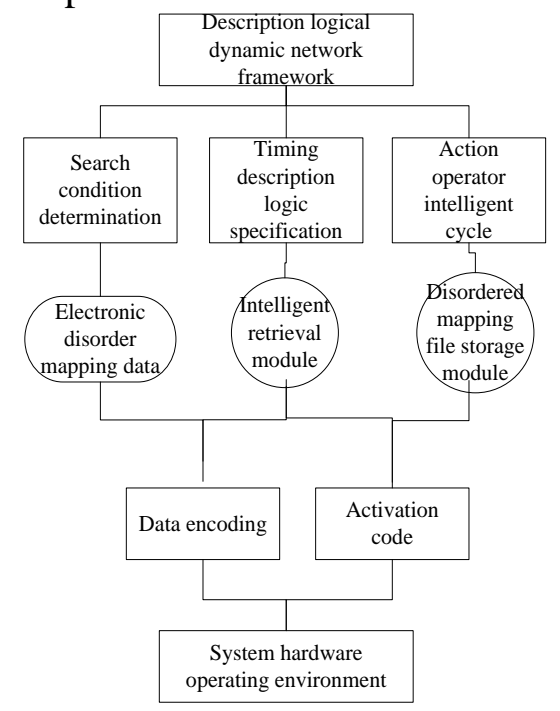

Figure 1 Automatic program dynamic network architecture structure

\subsection{Intelligent retrieval module design}

The new system intelligent retrieval module is an in-depth manifestation of the dynamic network framework timing description logic specification function. And under the promotion of the relevant decoding procedures, the coding data is activated and stimulated, so that the retrieval speed of all information is fully improved ${ }^{[6-7]}$. Within the scope allowed by the description logic, the upper limit value should be appropriately increased to avoid affecting the overall operating speed of the system ${ }^{[8-9]}$. The above steps are finished and the intelligent retrieval module construction is completed. The specific design principle is shown in Figure 2.

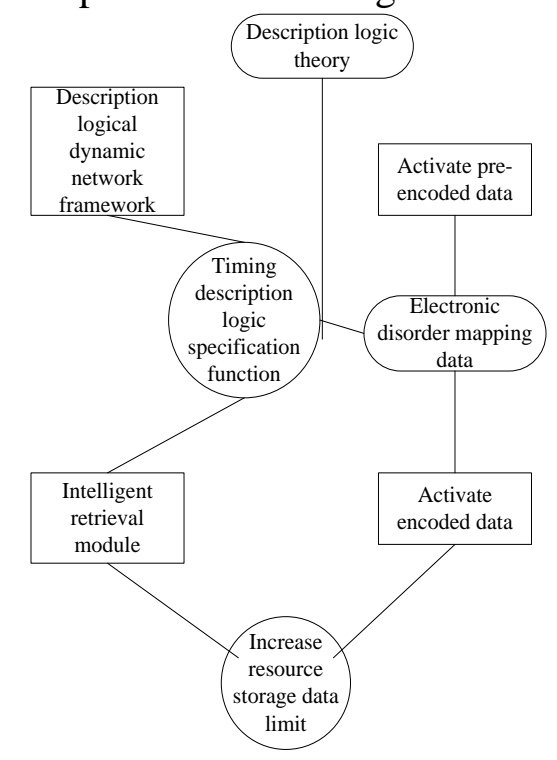

Figure 2 Intelligent retrieval module design schematic

\subsection{Disordered mapping file storage module design}

The working object of the disordered mapping archive storage module of the new intelligent retrieval system is mainly a series of multi-level architectures that conform to the automatic program relationship, such as media users. Under the influence of such changes, the system storage module also changes the monitoring method of the out-of-order mapping file data, which leads to the limitation of the total amount of resource storage, and the upper limit value gradually decreases 
with the increase of time ${ }^{[10]}$. According to the above theoretical principle, the design of the disordered mapping archive storage module is completed. The specific module structure is shown in Figure 3.

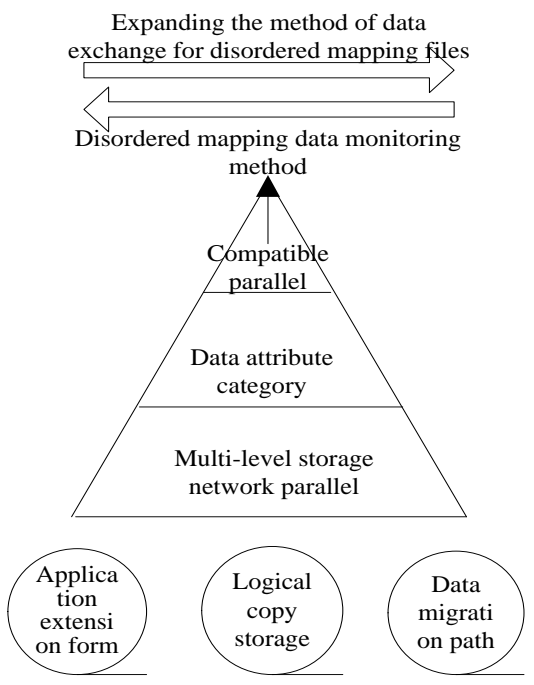

Figure 3 Schematic diagram of the disordered mapping archive storage module

\section{Software design of intelligent retrieval system for disordered surveying and mapping files}

On the basis of the system hardware operating environment, the three steps of retrieving the environment configuration, retrieval script design and retrieval process are performed to complete the construction of the system software running environment, and the smooth operation of the intelligent retrieval system based on the automatic program new disordered mapping and mapping system is realized.

\subsection{Intelligent disordered mapping and retrieval environment configuration}

The retrieval environment of intelligent disordered mapping files uses MyEclipsel0 as the basic research and development equipment, and realizes the real-time communication connection of lightweight mapping and archival data through Hibernate connection. Therefore, the configuration of the intelligent retrieval system of the new system intelligent disordered mapping is completed. The concrete construction principle is shown in Figure 4.

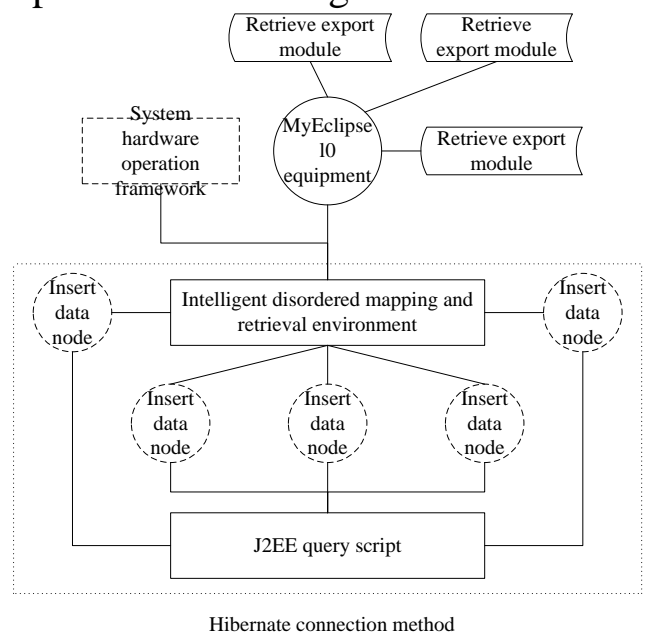

Figure 4 Schematic diagram of intelligent disordered surveying and mapping archive retrieval environment

\subsection{Automatic program query retrieval script design}

Under the constraint of description logic, the query retrieval script of the new system can deeply 
edit the insertion node of the data according to the Java language background. The Java scripting language uses the J2EE runtime environment as a dependency condition, and the programming code language becomes richer by continuously absorbing the way that the out-of-order mapping file retrieves and exports the module output instructions.

\section{Experimental results and analysis}

In order to verify the practical value of the new intelligent retrieval system based on automatic program, the following comparative experiments were designed. Two computers equipped with the same Java virtual machine were used as experimental objects, and the Tableau disordered mapping file retrieval system was used as a control group, and an intelligent retrieval system based on the automatic program new disordered mapping file was installed as an experimental group. 100 min was used as the experimental time and $15.0 \times 10^{11} \mathrm{~T}$ was used as the total amount of information retrieval.

\subsection{Experimental parameter setting}

The table below reflects the setting of relevant experimental parameters.

Table 1 Experimental parameter setting table

\begin{tabular}{|c|c|c|}
\hline Parameter name & Test group & Control group \\
\hline EPT/（min $)$ & 100 & 100 \\
\hline TIR/（T） & $15.0 \times 10^{11}$ & $15.0 \times 10^{11}$ \\
\hline LTS/ (T) & $\begin{array}{ll}9.7 \times 10^{11} & (\text { Peak }) \\
8.6 \times 10^{11} & (\text { Valley })\end{array}$ & $\begin{array}{ll}9.7 \times 10^{11} & \text { ( Peak }) \\
8.6 \times 10^{11} & \text { ( Valley })\end{array}$ \\
\hline DSP & $\begin{array}{l}0.75 \text { (Peak) } \\
0.49 \text { (Valley) }\end{array}$ & $\begin{array}{l}0.75 \text { (Peak) } \\
0.49 \text { ( Valley })\end{array}$ \\
\hline EIT/（min） & $\begin{array}{l}54.62 \text { ( Peak }) \\
69.74 \text { (Valley) }\end{array}$ & $\begin{array}{l}54.62 \text { (Peak) } \\
69.74 \text { (Valley) }\end{array}$ \\
\hline IEP & $\begin{array}{l}1.43 \text { ( Peak) } \\
1.13 \text { ( Valley) }\end{array}$ & $\begin{array}{l}1.43 \text { ( Peak }) \\
1.13 \text { ( Valley })\end{array}$ \\
\hline
\end{tabular}

The parameters in the above table represent the experimental time, the total amount of information retrieval, the upper limit of the target resource storage, the data storage parameters, the expected retrieval information export time, and the information export parameters.

\subsection{Resource storage cap comparison}

When the system is in the peak running state and the data storage parameter is 0.75 , taking $100 \mathrm{~min}$ as the experimental time, record the changes of the resource storage upper limit of the application experiment group and the control group system during the period of time. The specific experimental results are shown in Figures 5.

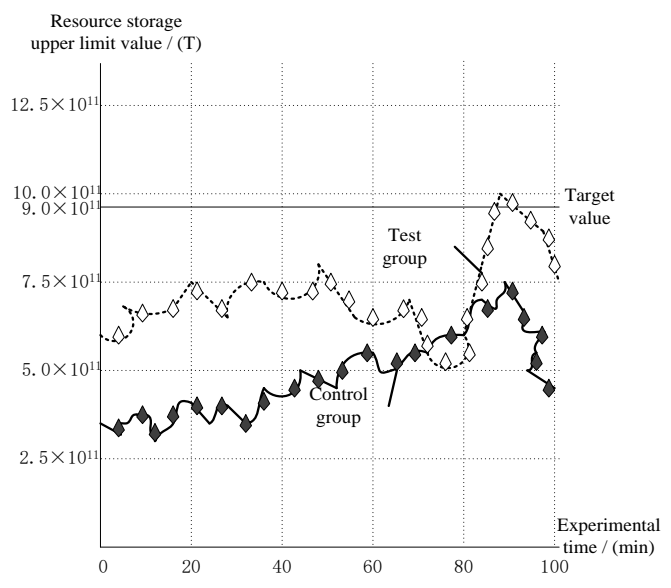

Figure 5 Resource storage upper limit comparison chart (peak operation status) 
Analysis of Figure 6 shows that when the system is in the peak running state, with the increase of running time, after applying the experimental group system, the upper limit of resource storage shows a trend of stability, decline, rise and fall.

\subsection{Search information export time comparison}

When the system is in the peak running state, when the information export parameter is 1.43 , using $15.0 \times 10^{11} \mathrm{~T}$ as the total amount of search information, the changes of the information export time after the application of the experimental group and the control system were recorded in the period of time. The specific experimental comparison results are shown in Table 2.

Table 2 Retrieval information export time comparison table (peak operation status)

$\begin{array}{llll}\begin{array}{l}\text { Retrieve } \\ \text { information }(/ \mathrm{T})\end{array} & \text { total } & \begin{array}{l}\text { Experimental group } \\ \text { retrieval information } \\ \text { export time (/ min) }\end{array} & \begin{array}{l}\text { Control information } \\ \text { retrieval time }(/ \mathrm{min})\end{array} \\ 3.0 \times 10^{11} & 30.20 & 55.48 \\ 6.0 \times 10^{11} & 30.20 & 55.48 \\ 9.0 \times 10^{11} & 30.20 & 55.48 \\ 12.0 \times 10^{11} & 30.20 & 55.48 \\ 15.0 \times 10^{11} & 30.20 & 55.48\end{array}$

Analysis of Table 2 shows that when the system is in the peak running state and as the running time increases, after applying the experimental group control system, the retrieval time of the search information remained stable, but the control group value was $55.48 \mathrm{~min}$, exceeding the target upper limit value of $54.62 \mathrm{~min}$, and the experimental group value was $30.20 \mathrm{~min}$, which was much lower than the experimental group.

\section{Conclusion}

Analysis and comparison of experimental results show that after applying the intelligent retrieval system based on the automatic disorder new mapping and mapping archives. Compared with the Tableau disordered mapping file retrieval system, the new intelligent retrieval system is simpler to construct, and the execution result is more accurate, which has strong practical promotion significance.

\section{References}

[1] Liu Jinhua, Li Lin, Liu Jianyu, Jia Shile. Design and application of residential electricity intelligent based on mobile internet and internet of things technology [J]. Electronic Design Engineering, 2017, 25(09): 157-161.

[2] Dong Qingchao, Chen Qinghua, Qi Yudong, et al. Contextual Environment Analysis Method of C4ISR System Based on Fuzzy Description Logic[J]. Systems Engineering and Electronics, 2016, 38(11): 2546-2551.

[3] Wu Jieqiong, Cai Qizhong, Pan Shaoming, et al. Design of small PLC logic operation controller based on ARM-FPGA[J]. Computer Engineering and Design, 2016, 37(9): 2394-2399.

[4] Sun Dayan, Xu Zufeng, Su Dawei, et al. Distributed Fault Diagnosis Based on Scheduling and Substation Integrated System[J]. Automation of Electric Power Systems, 2016, 40(23): 125-130.

[5] Ge Xiaobo, Shao Xiaodong, Qiu Yuanying, et al. A description of the semantics of product modeling rules based on framework model[J]. Journal of Computer Integrated Manufacturing Systems, 2016, 22(1): 232-240.

[6] Sun Jinyong, Qian Junyan, Tang Chenghua, et al. Case similarity of CBR based on fuzzy description logic[J]. Journal of Jinan University: Natural Science Edition, 2016, 30(5): 353-363. 
[7] Tai Yu, Mu Dejun, Hu Wei, et al. Research on multi-valued logic formalization method based on gate-level information flow analysis[J]. Journal of Northwestern Polytechnical University, 2017, 35(5): 884-889.

[8] Yuan Qinqin, Li Zhixun, Lü Lintao. Design and Optimization of Data Acquisition and Full Text Retrieval System Based on Oracle Components[J]. Modern Electronic Technology, 2016, 39(8): 3740 .

[9] Chen Wenbai, Huang Zhizhen, Liu Qiong. Design of a similar face retrieval system based on Pstabilized locally sensitive hash algorithm[J]. Journal of Intelligent Systems, 2017, 12(3): 392-396.

[10] Li Juan, Zhang Xiaobin, Xue Jiao, et al. Research on bamboo forest pest intelligent diagnosis system based on Lucid multi-path retrieval[J]. Journal of Zhejiang Agriculture and Forestry University, 2016, 33(1): 122-129. 